\title{
Respiratory Failure Secondary to Relapsing Polychondritis
}

\author{
Sridhar Badireddi MD, Mohammad F Siddiqui MD, and Neeraja J Boddu MD
}

\section{Introduction}

Diffuse airway narrowing of the trachea or main bronchus may result from various autoimmune connective tissue diseases. A careful clinical and pathological evaluation is warranted given the broad differential diagnosis. Relapsing polychondritis (RP) is a rare multisystem disorder that primarily targets cartilaginous structures; however, non-cartilaginous tissue invasion is also seen. Airway involvement by RP is not uncommon, and extra pulmonary manifestations are almost always the key to the diagnosis. Airway chondritis, diffuse tracheobronchial disease with tracheomalacia, and recurrent inflammation and infections as seen with RP are associated with poor prognosis. Hence, making an early diagnosis and providing prompt treatment in the early stages of the disease can change patient outcome. We report a case of a patient presenting with fever, cough, and RP who was diagnosed based on characteristic clinical and pathological findings.

\section{Case Summary}

A 65-y-old African American male presented with a 3-week history of fever, dry cough, ear and nose pain, and new onset hoarseness of voice. He had received 1 week of oral antibiotics for the treatment of community-acquired pneumonia and otitis externa. He denied nasal or ear discharge, skin rash, joint pain, nausea, vomiting, diarrhea, abdominal pain, hematuria, night sweats, chills, rigors, and appetite or weight

Drs Badireddi and Siddiqui are affiliated with the Division of Pulmonary and Critical Care Medicine, University of Arkansas for Medical Sciences, Little Rock, Arkansas. Drs Badireddi and Siddiqui are also affiliated with the Division of Pulmonary and Critical Care Medicine, and Dr Boddu is affiliated with the Division of General Internal Medicine, Central Arkansas Veterans Healthcare System, Little Rock, Arkansas.

The authors have disclosed no conflicts of interest.

Correspondence: Sridhar Badireddi MD, Division of Pulmonary and Critical Care Medicine, University of Arkansas for Medical Sciences, 4301 West Markham Street, Slot 555, Little Rock, AR 77205. E-mail: sbadireddi@gmail.com.

DOI: $10.4187 /$ respcare.02381 loss. On presentation, he was febrile at $38.2^{\circ} \mathrm{C}$ and tachypneic at 30 breaths $/ \mathrm{min}$, and he had a blood pressure of 105/70 mm Hg. Prominent physical findings included a flattened nasal bridge and swollen erythematous auricles that were tender to palpation (Fig. 1). A chest examination revealed diminished breath sounds in the left base with no adventitious sounds. A complete blood profile was significant for anemia, with hemoglobin of $8 \mathrm{~g} / \mathrm{dL}$ and leukocytosis of 27,000 white blood cells/ $\mu \mathrm{L}$. Arterial blood gas results revealed respiratory acidosis with $\mathrm{pH} 7.29, \mathrm{P}_{\mathrm{aCO}_{2}} 58 \mathrm{~mm} \mathrm{Hg}$, and $\mathrm{P}_{\mathrm{aO}_{2}} 84 \mathrm{~mm} \mathrm{Hg}$ on $2 \mathrm{~L} / \mathrm{min}$ oxygen. He was initiated on
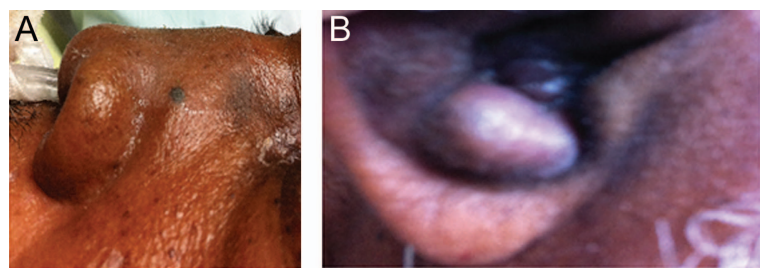

Fig. 1. A: Flattened nasal bridge (saddle nose deformity). B: Swollen and erythematous pinna and tragus of the left ear.

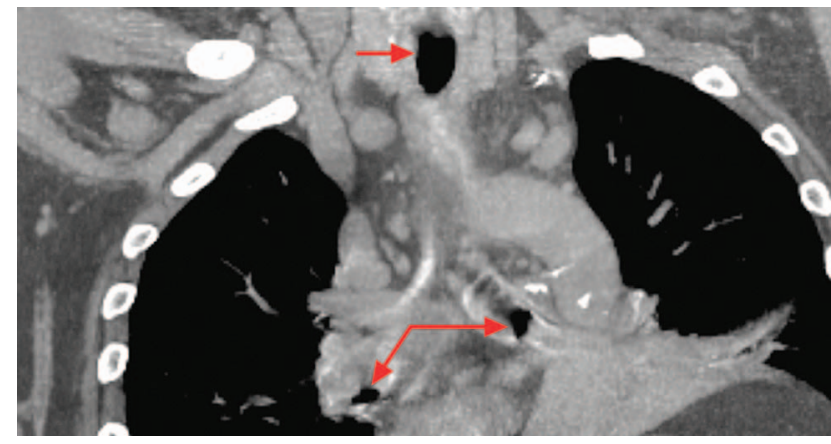

Fig. 2. Diffuse narrowing of the trachea and right and left main bronchus (arrows).
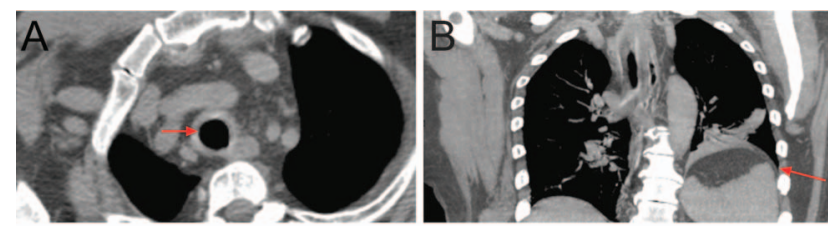

Fig. 3. Computed tomograms showing tracheobronchial wall thickening and hemidiaphragm elevation (arrows). 


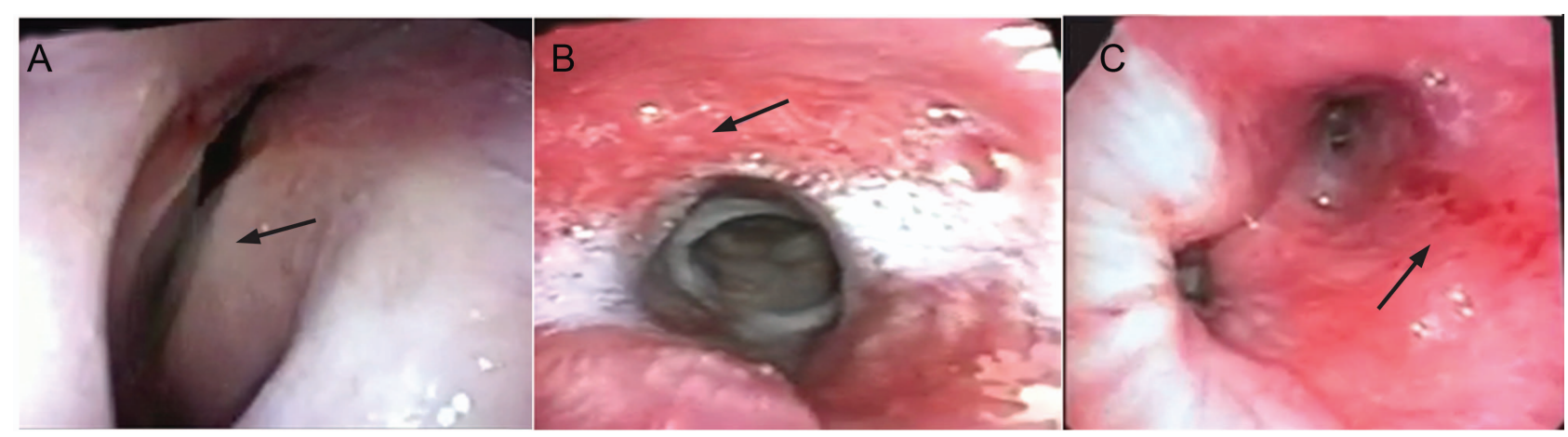

Fig. 4. A: Apposition of vocal cords during inspiration: paroxysmal vocal cord motion. B: Mucosal edema and erythema of the lower trachea and left main bronchus. C: Mucosal edema and erythema of the left lower lobe bronchus.
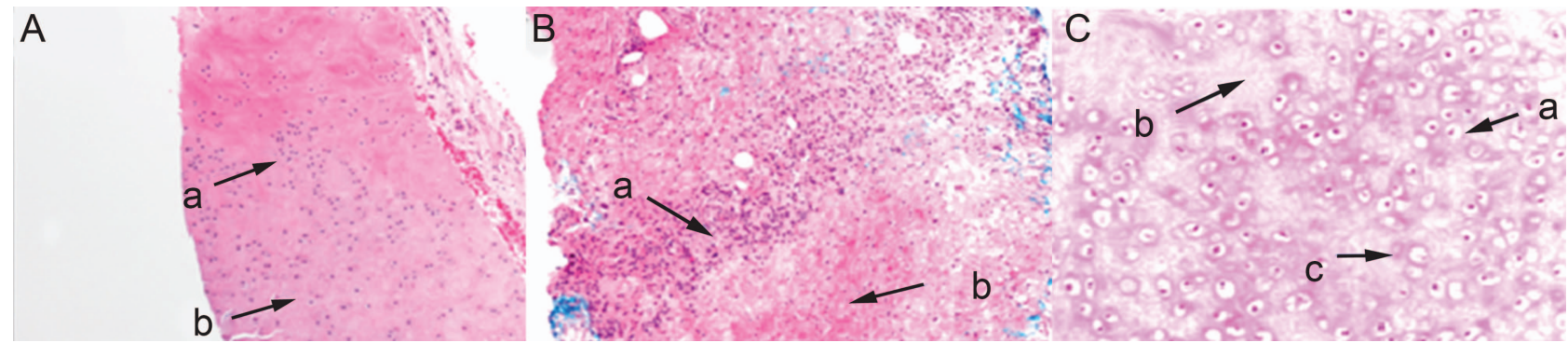

Fig. 5. A: Degenerated matrix (a) and normal matrix (b). B: Granulation tissue with fibroblasts (a) and degeneration of the matrix at the junction of granulation tissue and normal cartilage (b). C: Normal chondrocyte (a), degeneration of the matrix (b), and chondrocyte with area of necrosis (c).

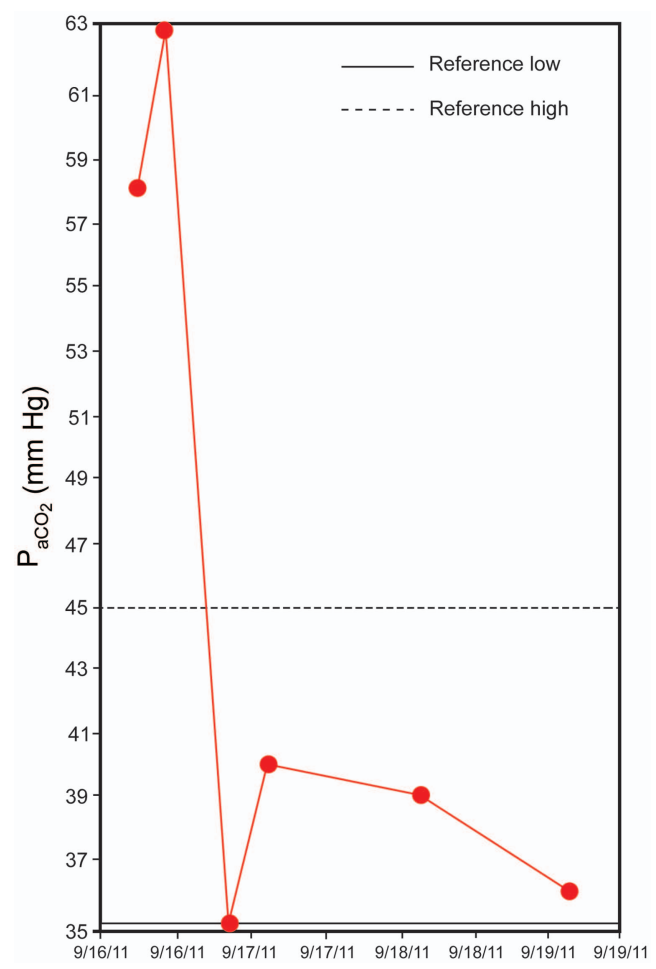

Fig. 6. Downtrend of $\mathrm{P}_{\mathrm{aCO}}$ after initiation of noninvasive ventilation. noninvasive ventilation at an inspiratory pressure of $10 \mathrm{~cm} \mathrm{H}_{2} \mathrm{O}$ and an expiratory pressure of $5 \mathrm{~cm} \mathrm{H}_{2} \mathrm{O}$ along with $2 \mathrm{~L} / \mathrm{min}$ oxygen. A computed tomography scan of the chest showed circumferential thickening of the trachea and an elevated left hemidiaphragm (Figs. 2 and 3).

Other relevant laboratory data included an elevated erythrocyte sedimentation rate of $120 \mathrm{~mm} / \mathrm{h}$ and C-reactive protein of $23.7 \mathrm{mg} / \mathrm{dL}$. The anti-neutrophil cytoplasmic antibody panel, anti-nuclear antibody, and rheumatoid factor were unremarkable. A flexible bronchoscopy performed with noninvasive ventilation revealed a paroxysmal vocal cord motion during inspiration (Fig. 4A), erythematous tracheal mucosa, and airway wall thickening of the left main bronchus (Fig. 4, B and C). An auricular biopsy showed areas of degenerated matrix and chondrocyte necrosis, with areas of granulation (Fig. 5). Based on the clinical presentation and histological findings, a diagnosis of RP was made. Treatment was initiated with prednisone at $60 \mathrm{mg} / \mathrm{d}$. The patient's symptoms, including fever, dyspnea, hoarseness of voice, and ear and nose pain, markedly improved within 1 week. There was also improvement in ventilation, with a gradual downtrend of $\mathrm{P}_{\mathrm{aCO}_{2}}$ (Fig. 6). Subsequently, the prednisone dose was gradually tapered over a period of 4 months. A repeat computed tomography scan of the chest at 4 months revealed resolution of tracheal wall thickness (Fig. 7). Five months after discontinuation of steroids, the patient had a relapse with an in- 


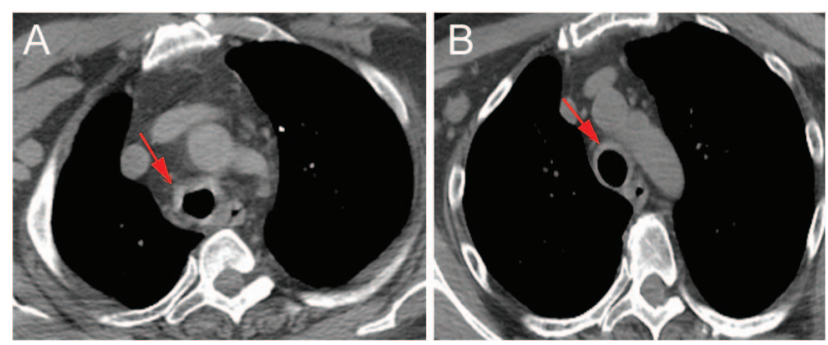

Fig. 7. A: Before prednisone therapy. B: Four months after prednisone therapy. Note the resolution of the circumferential thickness of the trachea after prednisone treatment (arrows).

creased erythrocyte sedimentation rate and C-reactive protein (Fig. 8). He was initiated on prednisone at $60 \mathrm{mg} / \mathrm{d}$ along with azathioprine at $100 \mathrm{mg} / \mathrm{d}$. He continued to have persistent respiratory symptoms with elevated inflammatory markers; hydroxychloroquine at $200 \mathrm{mg}$ twice daily was subsequently added to the regimen. The three-drug regimen was continued, with the patient demonstrating significant clinical improvement and reduced inflammatory marker levels.

\section{Discussion}

$\mathrm{RP}$ is a multisystem disease involving the ears and respiratory, musculoskeletal, skin, cardiovascular, renal, and neurological systems. McAdam et al $^{1}$ initially proposed a diagnostic criterion that has since been modified. Currently, the diagnosis is made if there are at least three of the following features: ocular inflammation; audiovestibular damage; non-erosive seronegative polyarthritis; and bilateral auricular, nasal, and laryngotracheal chondritis, along with histological confirmation and response to steroids/dapsone. ${ }^{2,3}$

Although commonly seen, airway involvement indicates poor prognosis if not diagnosed early. ${ }^{4}$ Involvement of larger airways such as the larynx and tracheobronchial tree is seen in almost half of patients with RP. Early bronchoscopy has a high diagnostic yield for diagnosing and preventing complications. ${ }^{5}$ The presenting symptoms can include hoarseness of voice, dysphonia, and respiratory distress. ${ }^{6}$ Cartilage inflammation leads to laryngeal and tracheal collapse with secondary infection. ${ }^{7}$ Apart from infection, a delay in diagnosing and treatment leads to tracheobronchomalacia and dynamic airway collapse.

Differential diagnosis of patients presenting with laryngotracheobronchial inflammation and narrowing should include granulomatosis with polyangiitis or Wegener's granulomatosis, amyloidosis, sarcoidosis, tracheopathia osteochondroplastica, and RP. ${ }^{8}$ In granulomatosis with polyangiitis, tracheal strictures, focal laryngeal stenosis, or cal-
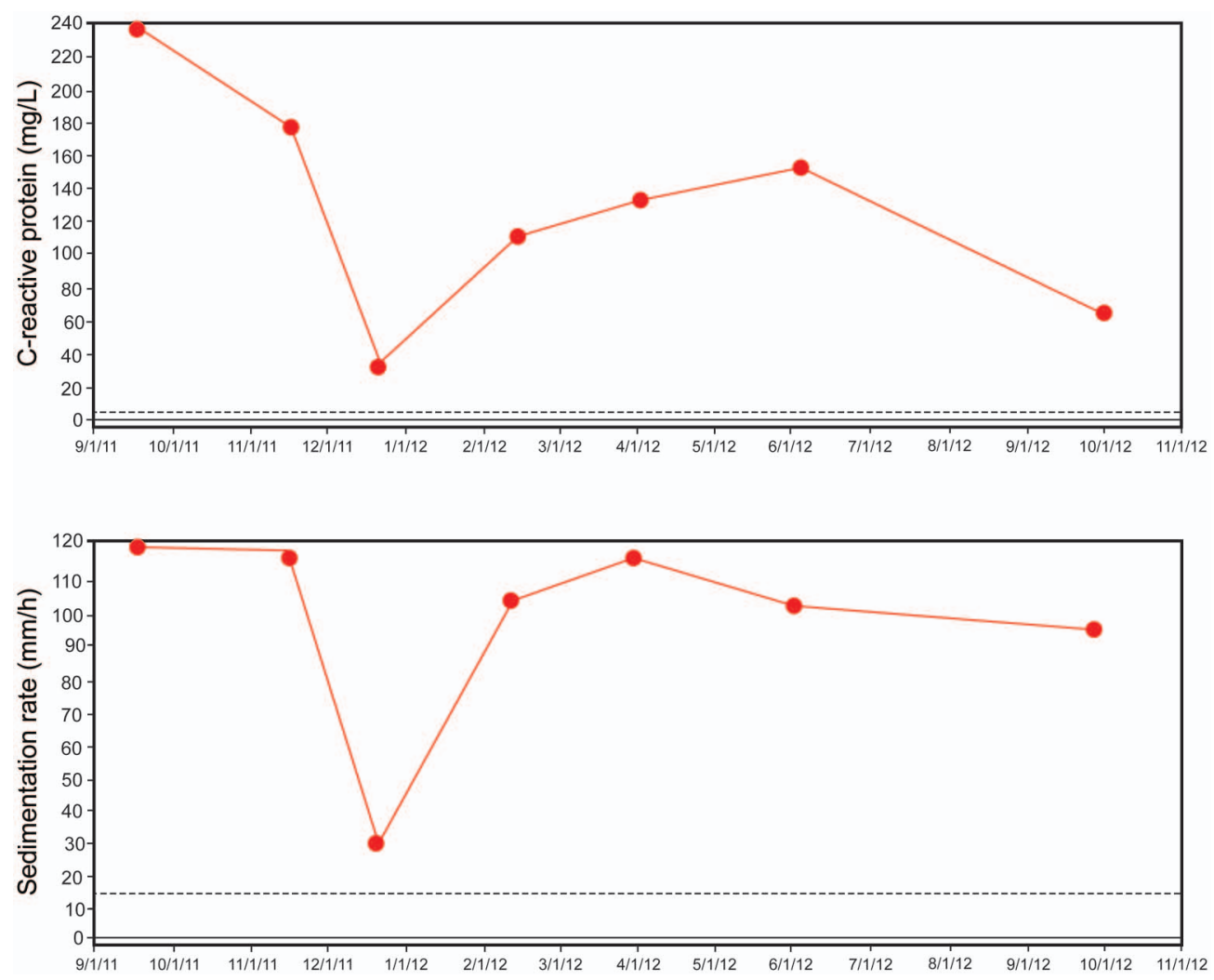

Fig. 8. Trends of erythrocyte sedimentation rate and C-reactive protein. The solid horizontal lines indicate reference low, and the dashed horizontal lines indicate reference high. 
cified tracheal rings are commonly seen. Tracheobronchial amyloidosis commonly manifests as diffuse mural thickening and luminal narrowing. Mural calcification may be evident on computed tomography scan. Endobronchial granulomata in patients with sarcoidosis may rarely result in significant airway narrowing. Tracheopathia osteochondroplastica is a rare, idiopathic, asymptomatic disorder of older men, characterized by multiple osteocartilaginous masses adjacent to the tracheal rings. This entity is distinguished from tracheobronchial amyloidosis and RP because it does not involve the posterior membranous portion of the trachea.

Diagnosis of RP is based mainly on clinical criteria, and a tissue biopsy may not be required. Some of the distinctive histological features of RP are loss of basophilic stippling of the cartilage matrix and perichondrial inflammation at the soft tissue-cartilage interface. Chondrocytes become vacuolated and necrotic and are eventually replaced by fibrous tissue.

Management of RP is largely empiric and based on case series and reports. No clinical trials have been performed due to the rarity of the disease. Airway management along with early pharmacotherapy can lead to relief of symptoms. ${ }^{2,7,9}$ Glucocorticoids, other immunosuppressive agents (such as methotrexate, cyclophosphamide, colchicine, and azathioprine), and anti-tumor necrosis factor agents have been used with varying outcomes ${ }^{10-16}$ Relapse of the disease is common when therapy is tapered off.

\section{Teaching Points}

- Cartilaginous structures are often involved in RP, and auricular involvement is a common feature.

- Airway inflammation is also commonly reported and portends poor prognosis if undiagnosed for a prolonged period of time.

- The pathogenesis of RP is unclear, and involvement of non-cartilaginous structures such as proteoglycan-rich organs like eyes and cranial nerves has been reported.

- Pharmacologic therapy is largely empiric and based on experience. Systemic steroids are the treatment of choice.
- Surgical interventions such as stent placement, localized tracheal resection, and tracheotomy are warranted in lifethreatening airway complications.

\section{REFERENCES}

1. McAdam LP, O’Hanlan MA, Bluestone R, Pearson CM. Relapsing polychondritis: prospective study of 23 patients and a review of the literature. Medicine 1976;55(3):193-215.

2. Ernst A, Rafeq S, Boiselle P, Sung A, Reddy C, Michaud G, et al. Relapsing polychondritis and airway involvement. Chest 2009;135(4): 1024-1030.

3. Michet CJ Jr, McKenna CH, Luthra HS, O'Fallon WM. Relapsing polychondritis. Survival and predictive role of early disease manifestations. Ann Intern Med 1986;104(1):74-78.

4. Damiani JM, Levine HL. Relapsing polychondritis-report of ten cases. Laryngoscope 1979;89(6 Pt 1):929-946.

5. Pearson CM, Kline HM, Newcomer VD. Relapsing polychondritis. N Engl J Med 1960;263:51-58.

6. Letko E, Zafirakis P, Baltatzis S, Voudouri A, Livir-Rallatos C, Foster CS. Relapsing polychondritis: a clinical review. Semin Arthritis Rheum 2002;31(6):384-395.

7. Tsunezuka Y, Sato H, Shimizu H. Tracheobronchial involvement in relapsing polychondritis. Respiration 2000;67(3):320-322.

8. Eng J, Sabanathan S. Airway complications in relapsing polychondritis. Ann Thorac Surg 1991;51(4):686-692.

9. Sarodia BD, Dasgupta A, Mehta AC. Management of airway manifestations of relapsing polychondritis: case reports and review of literature. Chest 1999;116(6):1669-1675.

10. Dunne JA, Sabanathan S. Use of metallic stents in relapsing polychondritis. Chest 1994;105(3):864-867.

11. Cazabon S, Over K, Butcher J. The successful use of infliximab in resistant relapsing polychondritis and associated scleritis. Eye 2005; 19(2):222-224.

12. Subrahmanyam P, Balakrishnan C, Dasgupta B. Sustained response to etanercept after failing infliximab, in a patient with relapsing polychondritis with tracheomalacia. Scand J Rheumatol 2008;37(3): 239-240.

13. Eng AM, Reddy VB. Relapsing polychondritis responding to colchicine. Int J Dermatol 1994;33(6):448-449.

14. Park J, Gowin KM, Schumacher HR Jr. Steroid sparing effect of methotrexate in relapsing polychondritis. J Rheumatol 1996;23(5):937938.

15. Hoang-Xaun T, Foster CS, Rice BA. Scleritis in relapsing polychondritis. Response to therapy. Ophthalmology 1990;97(7):892-898.

16. Vounotrypidis P, Sakellariou GT, Zisopoulos D, Berberidis C. Refractory relapsing polychondritis: rapid and sustained response in the treatment with an IL-1 receptor antagonist (anakinra). Rheumatology 2006;45(4):491-492. 